\title{
Modelling and design of an electrostatically levitated disc for inertial sensing applications
}

\author{
Michael Kraft, Mateen M Farooqui and Alan G R Evans \\ University of Southampton, Microelectronics Centre, Highfield, Southampton, SO17 1BJ, \\ UK \\ E-mail: m.kraft@soton.ac.uk
}

Received 5 January 2001, in final form 11 April 2001

\begin{abstract}
In this paper the design of a micromachined disc is described. The disc is encaged by electrodes at its top, bottom and periphery. Electrostatic forces are used to levitate the disc at the centre position. Such a device has applications for inertial sensors such as accelerometers and gyroscopes. It has considerable advantages over existing approaches, including increased independence of fabrication tolerances, online characteristics adjustment and in particular, compared to vibratory rate gyroscopes, inherently rules out quadrature error and the need for modal frequency tuning. A system level model of such a device is presented and the fabrication process relying on thick-film resist and electroplating is described.
\end{abstract}

(Some figures in this article are in colour only in the electronic version; see www.iop.org)

\section{Introduction}

Electrostatic levitation is a concept that has hardly been exploited for micromachined devices, although many potential applications exist, especially for inertial sensors, but also for micro-optical and microfluidic devices, frictionless bearings and micromotors $[1,2]$.

So far, micromachined accelerometers and gyroscopes typically use a proof mass which is connected to the substrate by a mechanical suspension system; this is subject to considerable manufacturing tolerances. Hence it is difficult to design a spring constant with an accuracy better than $\pm 10 \%$. Electrostatic forces may be used to introduce a negative spring constant and tune the overall spring constant; however, this requires considerable electronic circuitry and long-term drift effects may cause problems. Vibratory rate gyroscopes suffer from the problem of quadrature error and require precise frequency tuning between the excited drive and the sense mode, for which, to date, no satisfactory solution has been presented [3].

In this paper the design of a micromachined disc levitated by electrostatic forces is described which can be used for inertial sensing applications and to overcome some of the aforementioned problems. Previous work in this direction has mainly made use of electromagnetic forces [4] and resulted in a levitated disc which was not encaged. Other researchers reported a levitated disc with electrostatic forces; however, the chosen control approach requires inductors which are not suitable for integrated electronics [5]. The design presented in this paper does not have these disadvantages. Here the envisaged application is for acceleration sensing to prove the feasibility of the approach. At a later stage the disc will be spun about its main axis and be used as a gyroscope. The disc is encaged by electrodes on each side to which voltages are applied in such a way that the disc is levitated at the centre position parallel to the substrate. Measurements of the capacitances formed between the electrodes and the disc can be used to determine the position of the disc. The position information is used to generate feedback voltages on the electrodes which produce a net electrostatic force on the disc and cancel all external inertial forces. The required feedback voltages also provide a measure of acceleration. Since the effective spring constant merely depends on the applied electrostatic forces, it can be adjusted by simply changing the applied feedback voltages. The sensitivity and bandwidth of the sensor can thus be changed to suit the required application. The system is shown schematically in figure 1 .

\section{Control strategy}

The control strategy is based upon the incorporation of the mechanical sensing element in a sigma-delta modulator control 
Clocked

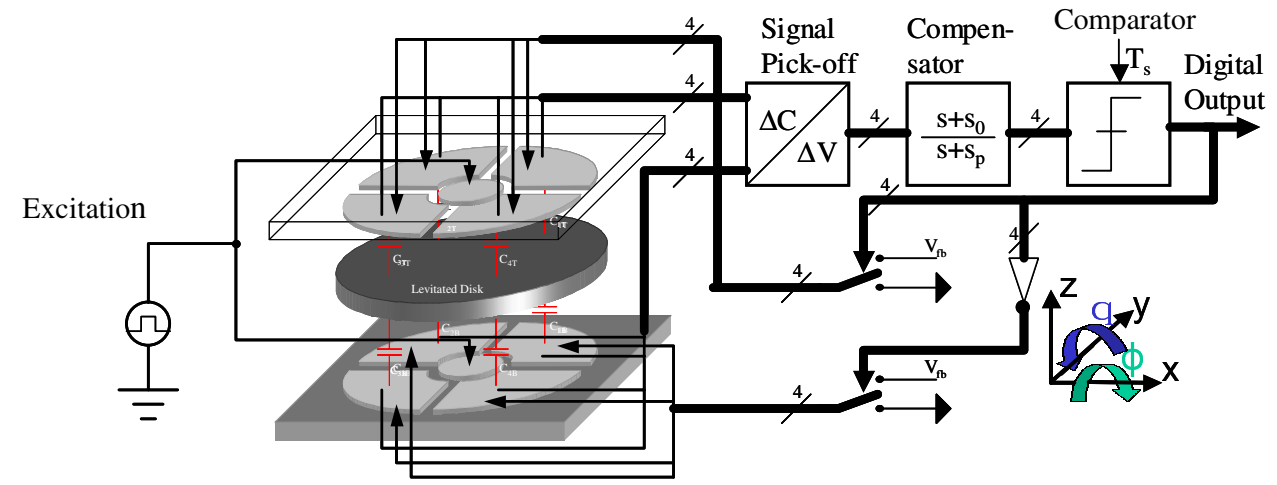

Figure 1. Levitated disc and control system (electrodes at the periphery of the disc are not shown).

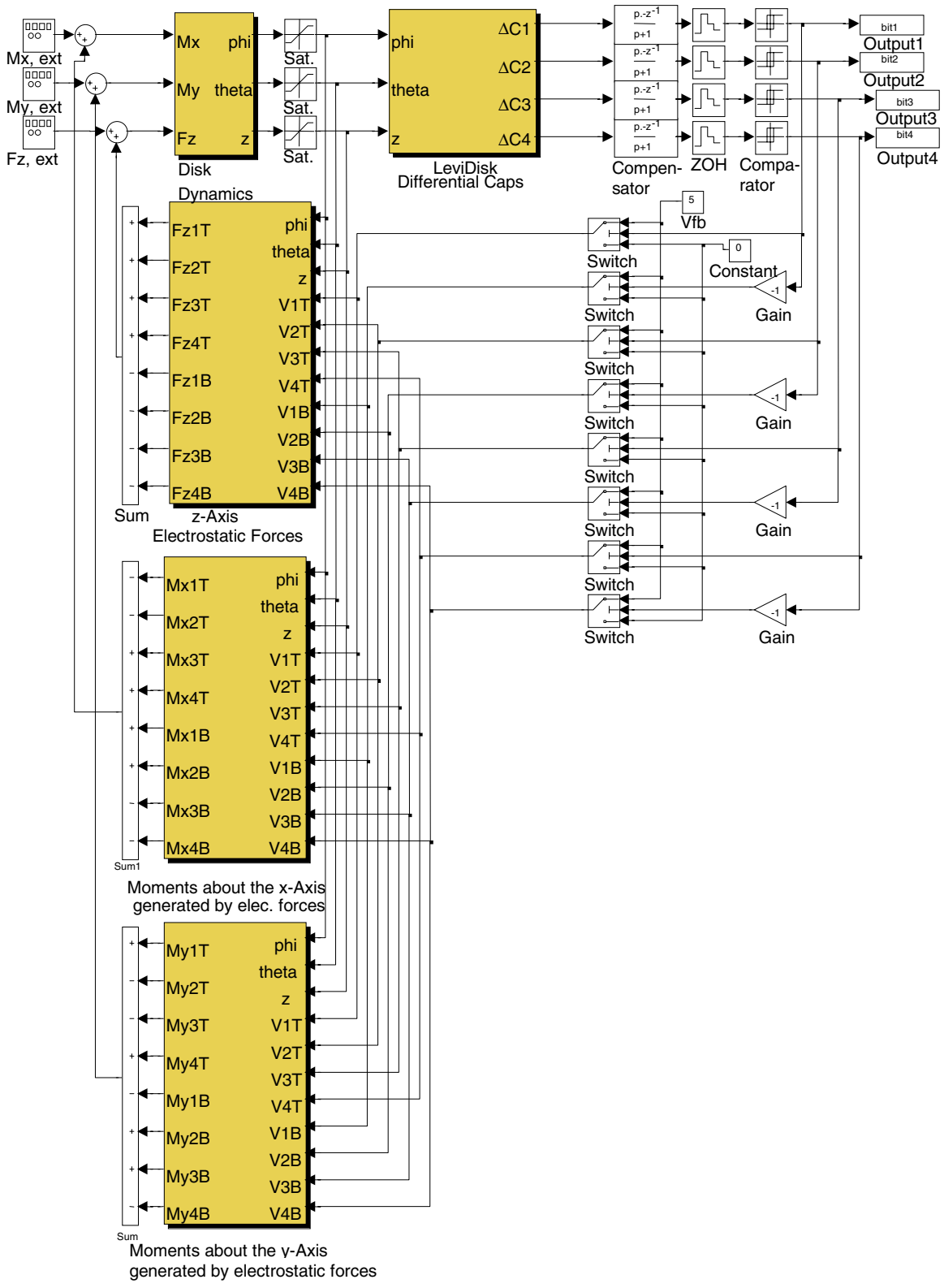

Figure 2. Top level Simulink model of the levitated disc. 
system (see figure 1). This has several advantages over analogue control systems, such as increased system stability, a direct digital output signal in the form of a pulse density modulated bitstream, and it also retains the usual merits of closed-loop control, such as an increase in bandwidth, dynamic range and linearity [6]. The control system comprises four sigma-delta modulator channels, one for each differential capacitor. A clocked comparator is used to determine the sign of the differential capacitance which, in turn, contains the information on whether the disc is closer to the top or bottom electrode. The comparator controls a switch for each electrode, determining which electrode will be energized. The output signal of the comparator provides a pulse density modulated, serial bitstream and can be directly processed further by digital signal processing algorithms. In-between the signal pick-off and the comparator a compensator is required which stabilizes the loop by adding phase lead.

\section{Simulation}

A system level model has been derived comprising the following building blocks: disc dynamics, capacitive position measurement interface, compensator, sigma-delta modulator building blocks, electrostatic feedback forces and moments on the disc. The model was implemented in a system level simulator; here Matlab/Simulink was chosen since this tool is not domain specific, hence it is easy to simulate a system consisting of mechanical and electronic parts. It allows the optimization of crucial system parameters such as the disc geometry, sampling frequency, feedback voltage, etc. The full model is shown in figure 2. The input signals to the model are the external inertial forces and moments. The building blocks of the disc dynamics calculate the position of the disc from which, in turn, the differential capacitors for each of the four outer electrodes are calculated. This signal is then passed on to the building blocks of the sigma-delta modulator. The state of the clocked comparators control a switch for each outer electrode which either applies a feedback voltage to the electrode or grounds it. From the potential applied to the eight feedback electrodes the electrostatic force in the $z$-direction and the moments about the $x$ - and $y$-axes are calculated. The derivation of the analytical equations for the model is described in detail in [7]. The disc acts as a summing element for the forces and moments.

One fundamental difference between a conventional accelerometer and the levitated disc approach is the lack of a mechanical spring force. As a consequence the dynamics of the sensing element comprise the characteristics of an ideal integrator, whereas a mechanical spring constant has the equivalent effect as an integrator with leakage which increases the quantization noise at low frequencies in sigma-delta modulators [8]. The model in its present form only captures the governing features of the device, nevertheless it already allows optimization of the choice of important parameters, such as geometry, feedback voltages and sampling frequency, and also aids in the design of the control strategy. The simulation results are based upon a disc with a diameter of $1 \mathrm{~mm}$, a thickness of $100 \mu \mathrm{m}$, gaps to the electrodes on either side of $2 \mu \mathrm{m}$, feedback voltages of $20 \mathrm{~V}$ and a clock frequency of $1 \mathrm{MHz}$. Figure 3 shows the steady-state condition for the pulse

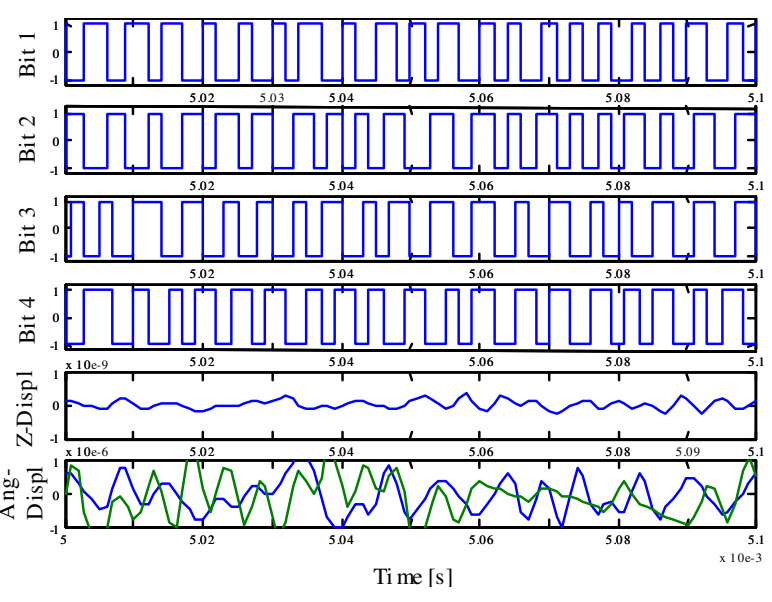

Figure 3. Output pulse density modulated bitstreams in the unforced steady-state condition (upper four waveforms). The two lower waveforms show the residual movement of the disc in the $z$-direction and the two tilting angular motions about $x$ - and $y$-axes, respectively.
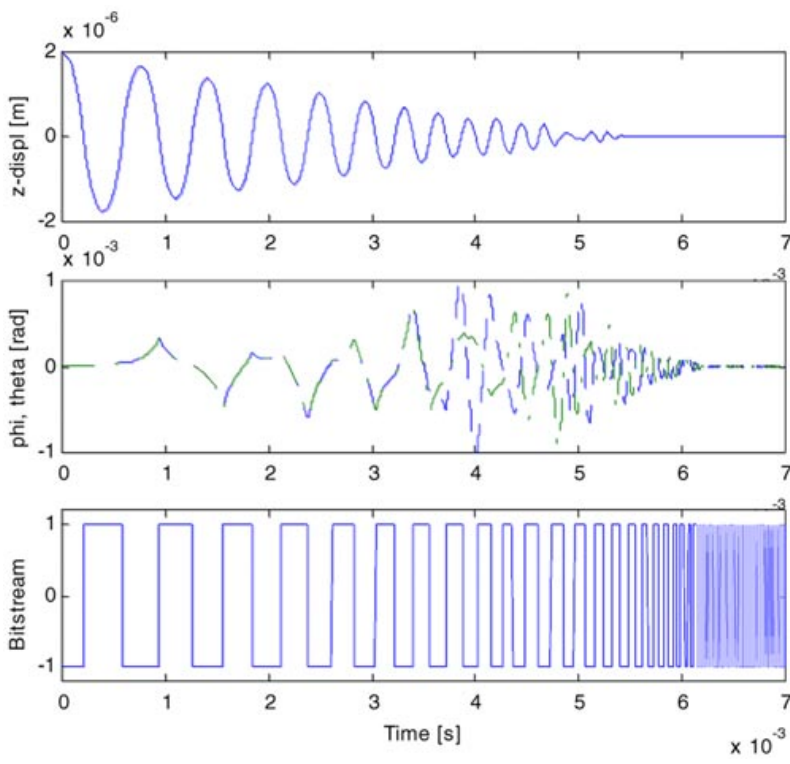

Figure 4. Simulation result showing the start-up phase. The disc reaches the middle position after approximately $7 \mathrm{~ms}$. Upper plot, displacement of the disc in the $z$-direction; middle plot, tilt of the disc about the $x$-axis (dashed) and $y$-axis (dash-dot); and bottom plot, one of the four output bitstreams.

density modulated bitstreams in the upper four diagrams. The exhibited waveforms indicate a limit cycle frequency which alternates between one quarter of the sampling frequency $f s$ (a $(2,2)$ mode) and $f s / 6(\mathrm{a}(3,3)$ mode $)$ which is the expected behaviour for a second-order system [6]. The lower two waveforms show the residual motion of the proof mass in the $z$-direction and the tilting angular motion about the two inplane axes. These residual movements are much smaller than the gap, indicating sufficient gain in the control system and validating the assumption of negligible nonlinear behaviour due to squeeze film damping effects. Figure 4 shows a simulation run of the start-up phase in which the disc initially lies on one set of electrodes (i.e. an initial $z$-displacement of $2 \mu \mathrm{m})$; after a transient behaviour the control system captures 


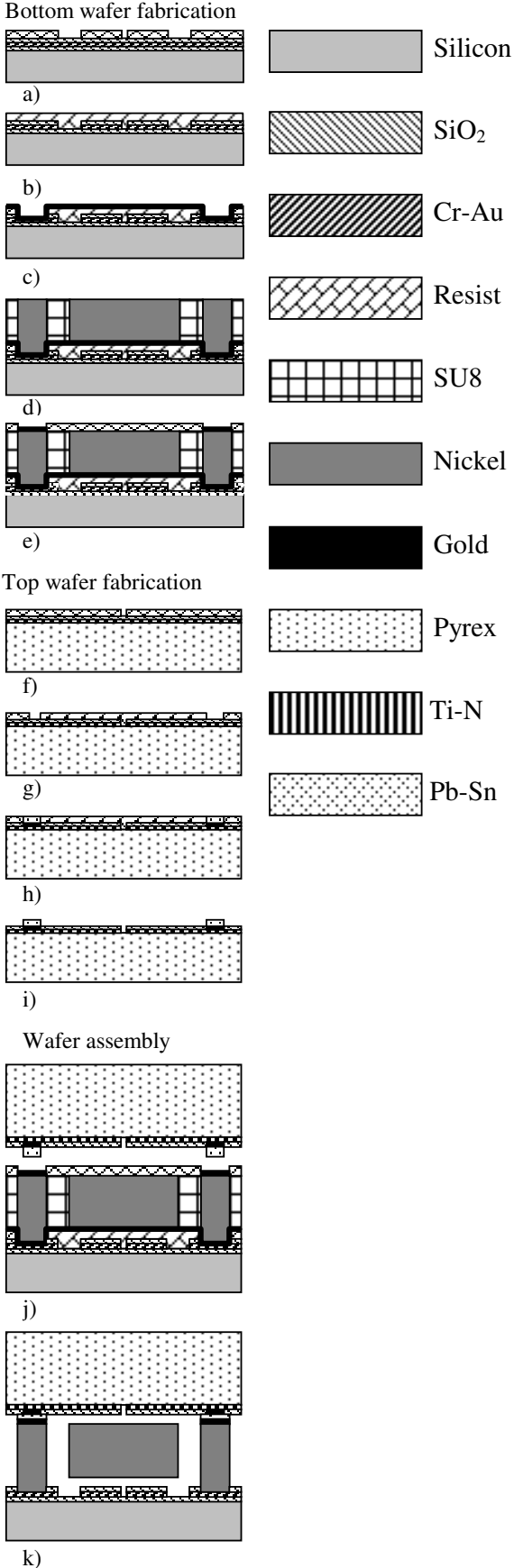

Figure 5. Process flow: $(a)-(e)$ bottom wafer fabrication, $(f)-(i)$ top wafer fabrication and $(j)-(k)$ assembly. For details see the main text.

the disc and ensures that it is moved to the mid-position between the electrodes. Effects which are difficult to describe analytically such as squeeze film damping, heat sensitivity and fringing fields will be simulated in MEMCAD.

\section{Fabrication}

A suitable fabrication process is under development. A fundamental question is whether to use thin-film, i.e. surface micromachining, or thick-film technology. Previous work that used thin films as a material for making a levitated disc [2] suffered from the disadvantage that mechanical

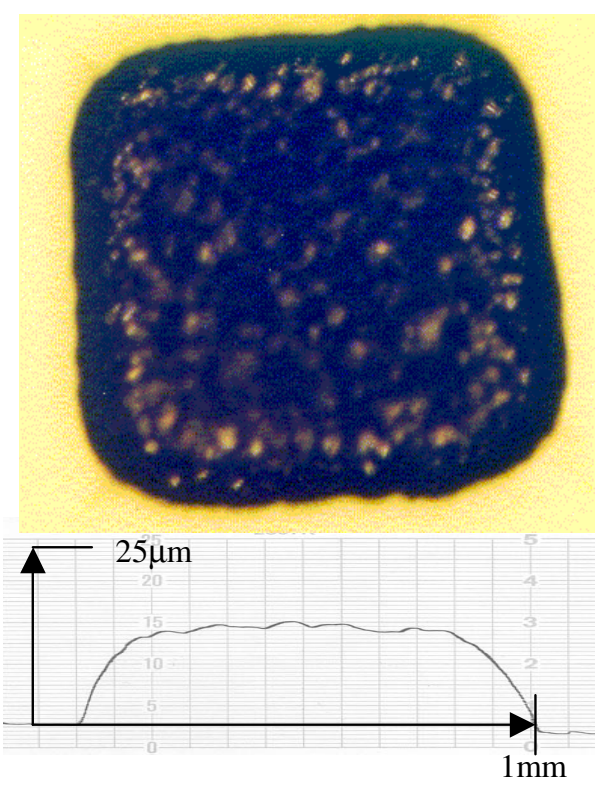

Figure 6. Electroplated structure and its profile, showing the 'mushrooming' effect.

eigenmodes of the disc may be excited by the feedback forces and moments [9]. Consequently, we decided to fabricate a relatively thick disc (in the range of several hundreds of micrometres) which has the further advantage that the sensitivity to inertial forces is higher.

The device will be fabricated by a process relying on electroplating into a mould of a special thick film resist (SU8), which is compatible with standard processing for integrated circuits. However, for simpler prototype evaluation, discrete off-chip electronics will be developed. The assembled device consists of two wafers bonded together. On the bottom wafer a $1 \mu \mathrm{m}$ wet oxide is grown, $\mathrm{Cr}-\mathrm{Au}$ is evaporated and another layer of oxide is deposited by LPCVD. Both the oxide and the $\mathrm{Cr}-\mathrm{Au}$ layers are dry-etched to the pattern of the bottom electrodes (figure 5(a)). In the next step the spacer resist is spun on; this is a crucial step since it later defines the gap between the disc and the lower electrodes (figure 5(b)). Subsequently, the resist is patterned with the mask for the pillars which encage the disc and also provide the electrical connection to the top electrodes. Then a thin layer of $\mathrm{Au}$ is evaporated, which acts as the seed layer for the electroplating step (figure 5(c)). This is followed by spinning on the SU8 resist, which can form layers up to $500 \mu \mathrm{m}$ thick. The SU8 is then patterned with the mask defining the pillars and the disc. On the Au seed layer nickel is electroplated, forming the disc and the pillars. Since it is very difficult to stop the plating at the right moment in time, some 'mushrooming' is usually observed, as shown in figure 6 . This is removed by a diamond lapping and polishing step (figure $5(d)$ ). Next, the upper spacer resist is spun on, patterned with the mask for the pillars and a thin layer of Au is plated on the nickel. This is the final step for the first wafer (figure 5(e)).

The top electrodes are formed by evaporating $\mathrm{Cr}-\mathrm{Au}$ (or $\mathrm{Ti}-\mathrm{N}$ because it is transparent) on a Pyrex wafer, then oxide is deposited by PECVD. Both layers are etched to define the top electrodes (figure $5(f)$ ). The resist is spun on and patterned with the mask for the pillars (figure $5(g)$ ), subsequently $\mathrm{Au}$ 
is evaporated as a seed layer to electroplate $\mathrm{Pb}-\mathrm{Sn}$, which is used as a solder to bond the two wafers together (figure 5(h)). Finally, the resist layer is removed (figure 5(i)). The two wafers are bonded together by eutectic reflow of the $\mathrm{Pb}-\mathrm{Sn}$ solder (figure $5(j)$ ). In the last step the SU8 is removed by plasma ashing and the disc is freed (figure $5(k)$ ).

Currently, batches of the first prototypes are being fabricated. The masks will contain arrays of varying geometries for disc diameter (from $100 \mu \mathrm{m}$ to $2 \mathrm{~mm}$ ), disc thickness (from $50 \mu \mathrm{m}$ to $500 \mu \mathrm{m}$ ) and spacer resist (from $2 \mu \mathrm{m}$ to $5 \mu \mathrm{m}$ ). Experimental results on the process and measurement data will be published in a following paper.

\section{Conclusions}

Electrostatic levitation is a relatively novel approach to inertial sensing which has many applications. In this paper the design of a prototype is described which will lead to a multi-axis accelerometer having the advantages that its effective spring constant is solely determined by electrostatic forces resulting from applied voltages. Additional electrodes will be used to make the disc spin and demonstrate its application as a gyroscope. The system level model described proved to be a valuable tool for design and to verify the feasibility of the concept.

\section{References}

[1] Fukatsu K, Murakoshi T and Esashi M 1999 Electrostatically levitated micro motor for inertia measurement system Transducer '99 3P2.16

[2] Torti R, Gondhalekar V, Tran H and Selfors B 1994 Electrostatically suspended and sensed micro-mechanical rate gyroscope Proc. SPIE 2200 27-38

[3] Clark W A, Howe R T and Horowitz R 1996 Surface micromachined Z-axis vibratory rate gyroscope Solid State Sensors and Actuator Workshop pp 283-7

[4] Shearwood C, Williams C B, Mellor P H, Yates R B, Gibbs M R J and Mattingley A D 1995 Levitation of a micromachined rotor for application in a rotating gyroscope Electron. Lett. 31 1845-6

[5] He G, Chen K, Tan S and Wang W 1996 Electrical levitation for micromotors, microgyroscopes and microaccelerometers Sensors Actuators A 54 741-5

[6] Kraft M, Lewis C P and Hesketh T G 1998 IEE Closed loop silicon accelerometers Proc. IEE Circuits, Devices Syst. 145 $325-31$

[7] Kraft M and Evans A 2000 System level simulation of an electrostatically levitated disk Proc. 3rd Conf. on Modeling and Simulation of Microsystems (San Diego, CA, March 2000) pp 130-3

[8] Candy J C and Temes G C (eds) 1992 Oversampling Delta-Sigma Converters (New York: IEEE Press) pp 1-29

[9] Handtmann M, Aigner R, Ploetz F and Wachutka G 1999 Proc. SISPAD'99 (Kyoto, Japan, 1999) pp 183-6 